\section{Endoscopic Appearance of Malignant Melanoma Metastatic to the Upper Gastrointestinal Tract}

Only a few reports of endoscopic findings in patients with metastatic lesions in the gastrointestinal (GI) tract arising from a malignant melanoma are available $(1-3)$. We therefore report two such patients who were diagnosed using endoscopy.

A 50-year-old man presented with epigastric pain after meals, anorexia, weight loss, and back pain suggestive of root pains for one year. Right submental lymph node enlargement, and deformity and tenderness of thoracic spine were present. X-rays revealed mediastinal lymphadenopathy, multiple bilateral pulmonary metastases and destruction of the body and pedicles of the eighth dorsal vertebra with preserved disc spaces.

An upper Gil endoscopy revealed an ulcer 2-cm in diameter high on the lesser curve with an irregular friable and ulcerated surface with areas of blackening (Figure 1 a) and multiple, 3-6 mm diameter, black, well-circumscribed elevated areas in the gastric fundus, antrum, and duodenal bulb (Figure 1 b). Biopsies from these lesions revealed malignant melanoma. A barium meal study revealed a rounded filling defect with a central pool of contrast (target sign) on posterior wall of the gastric corpus. A review of the patient's history revealed that he had had three teeth and part of his right lower gum removed for what was described as a black lesion involving this region.

An 85-year-old man presented with post-prandial epigastric pain, anorexia, weight loss, and an enlarging hard left inguinal lymph node mass for two months. Fine needle cytology of the mass revealed spindle-shaped cells that contained abundant cytoplasm. brownish black pigment, and anisomorphic nuclei with few nucleoli suggestive of malignant melanoma. An upper Gl endoscopy revealed multiple discrete, well-demarcated, elevated black nodular lesions in the lower esophagus (Figure 2), the stomach, and the second and third parts of the duodenum. Biopsies from these lesions confirmed these to be metastases from a malignant melanoma.

Malignant melanoma metastasizes frequently to the upper Gl tract. The overall frequency of gastric metastases from a melanoma is $10-20 \%(1,2)$. However, an endoscopic diagnosis of metastatic malignant melanoma is rare $(1-3)$. Black nodules with a central necrotic tip, are the most common endoscopic finding. Melanin is present in most cases, but occasionally the lesions can be amelanotic (3). Biopsies are positive in $92-100 \%$ of cases (2). Both our patients had multiple nodular lesions in the stomach and the duodenum; in addition the esophagus was involved in one case. In one patient, some lesions also showed central necrosis and ulceration. Biopsies were positive in both the patients. Awareness of endoscopic findings in cases of metastatic malignant melanoma may be important if the primary lesion is insignificant or has been forgotten, as exemplified by our first case.

R. Gupta, R. Aggarwal, A. S. Puri, S. Shukla, R. Pandey, S. R. Naik Departments of Gastroenterology and Pathology, Sanjay Gandhi Postgraduate Institute of Medical Sciences, Lucknow, India

\section{References}

1. Shah SM, Smart DF, Texter EC, et al.: Metastatic melanoma of stomach: endoscopic and roentgenographic findings. South Med J 1977: 70: 379-382.

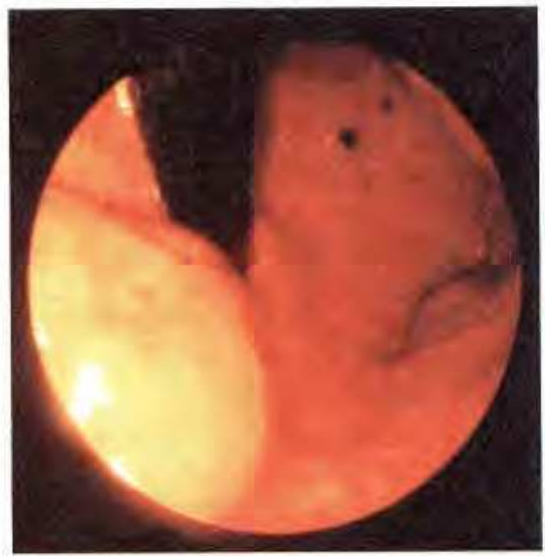

Figure 1 a: An uker high on the lesser curve of the stomach showing irregularity, ulceration, friability, and areas of blackening.

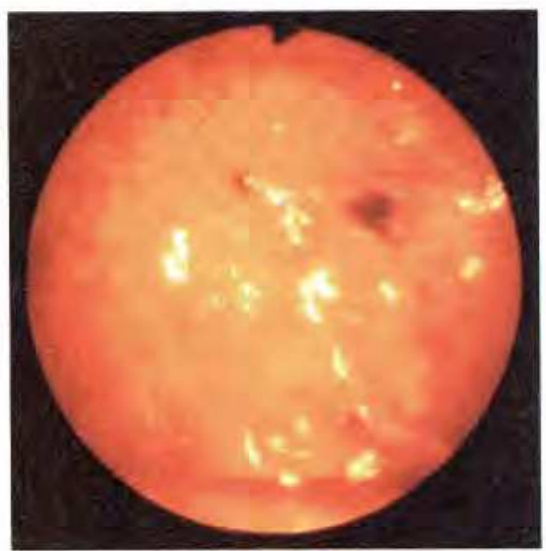

Figure 1 b: Metastases from a malignant melanoma in the form of multiple. elevated, nodules with black pigmentation in the stomach.

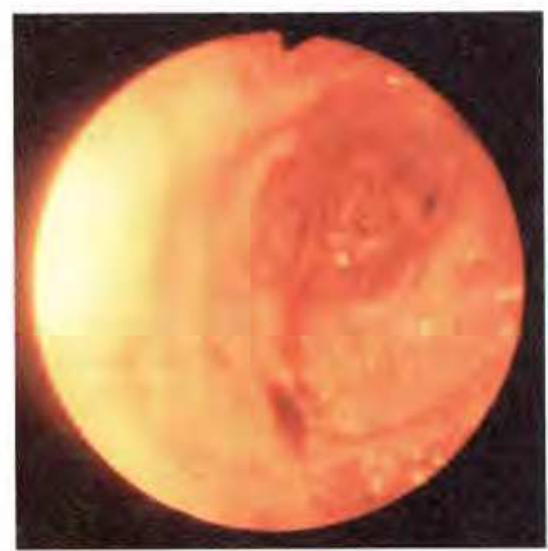

Figure 2: Multiple, elevated, black, nodu lar lesions in the lower esophagus.

2. Nelson RS, Lanza F: Malignant melanoma metastatic to the upper gastrointestinal tract. Gastrointest Endosc 1978; 24: 156 158.

3. Kadakia SC, Allan Parker DO, Canales L: Metastatic tumors to the upper gastrointestinal tract: endoscopic experience. Am J Gastroenterol 1992; 87: 1418-1423.

\section{Corresponding Author}

R. Aggarwal, M.D.

Department of Gastroenterology

Sanjay Gandhi Postgraduate Institute of Medical Sciences

Luckow 226014

India 